\title{
STABLY FREE RESOLUTIONS OF LATTICES OVER FINITE GROUPS
}

\author{
K. W. GRUENBERG
}

(Received 2 June 1989)

Communicated by H. Lausch

Dedicated to Tim Wall, in recognition of his distinguished contribution to mathematics in Australia, on the occasion of his retirement

\begin{abstract}
For a $\mathbb{Z} G$-lattice $A$, the $n$th partial free Euler characteristic $\varepsilon_{n}(A)$ is defined as the infimum of all

$$
\sum_{i=0}^{n}(-1)^{n-i} d_{G}\left(F_{i}\right)
$$

where $F_{*}$ varies over all free resolutions of $A$. It is shown that there exists a stably free resolution $E_{*}$ of $A$ which realises $\varepsilon_{n}(A)$ for all $n \geq 0$ and that the function $n \rightarrow \varepsilon_{n}(A)$ is ultimately polynomial on residue classes. The existence of $E_{*}$ is established with the help of new invariants $\sigma_{n}(A)$ of $A$. These are elements in certain image groups of the projective class group of $\mathbb{Z} G$. When $\mathbb{Z} G$ allows cancellation, $E_{*}$ is a minimal free resolution and is essentially unique. When $A$ is periodic, $E_{*}$ is ultimately periodic of period a multiple of the projective period of $\boldsymbol{A}$.
\end{abstract}

1980 Mathematics subject classification (Amer. Math. Soc.) (1985 Revision): 20 C 10, 20 C 05, $18 \mathrm{G} 10$.

\section{Introduction}

Our aim in this paper is to compare the projective and free resolutions of a lattice $A$ over $\mathbb{Z} G$, the integral group ring of a finite group. An example where such a comparison has some importance is when $A$ is periodic: this means that there exists a projective resolution which repeats with some period

(C) 1990 Australian Mathematical Society 0263-6115/90\$A2.00+0.00 
$q$. Does $A$ then have a periodic free resolution also of period $q$ ? The answer depends on the vanishing of a particular element in a certain factor group of the projective class group of $\mathbb{Z} G$. This was discovered many years ago in the special case $A=\mathbb{Z}$ by Swan [4]. We shall show here that his ideas, supported by additional material, lead to similar results in general.

A numerical measure of the difference between projective and free resolutions is given by the two partial Euler characteristics introduced by the author in [2]. It was proved there that the partial projective Euler characteristics $\chi_{n}(A), n \geq 0$, are realised by any minimal projective resolution of $A$. Moreover, the associated Poincaré series $\sum_{n \geq 0} \chi_{n}(A) t^{n}$ is rational of the form $g(t) /\left(1-t^{q}\right)^{m}$, where $g(t) \in \mathbb{Z}[t]$ and $m, q \in \mathbb{Z}>0$. This was observed by P. Webb [7] and depends on earlier work of Evens and Swan which enables one to calculate (in principle) the function $\chi(A)$ in terms of data from the modular representation theory of $G$. No such method of calculation seems available for the partial free Euler characteristics $\varepsilon_{n}(A), n \geq 0$. Nevertheless, by adopting an indirect approach, we shall prove here that $\sum_{n \geq 0} \varepsilon_{n}(A) t^{n}$ is rational of the same form as the $\chi(A)$-series. There is, in general, no free resolution that realises $\varepsilon(A)$, but we establish the existence of a stably free resolution that does. When $\mathbb{Z} G$ allows cancellation, this is a minimal free resolution and then, by a result in [3], every minimal free resolution of $A$ realises $\varepsilon(A)$.

The functions $\chi(A)$ and $\varepsilon(A)$ are related by inequalities

$$
\frac{1}{|G|} \chi_{n}(A) \leq \varepsilon_{n}(A) \leq \frac{1}{|G|} \chi_{n}(A)+1 \text {. }
$$

The proof of our stably free resolution theorem depends on relating the set of integers $n$ where the first of these inequalities is an equality with the vanishing of invariants $\sigma_{n}(A)$ that live in different image groups of the projective class group of $\mathbb{Z} G$ and generalise the Swan invariant for $\mathbb{Z}$ mentioned earlier. When $A$ is a non-periodic lattice, $\sigma_{n}(A)=0$ for all sufficiently large $n$ and consequently the functions $\varepsilon(A)$ and $\frac{1}{|G|} \chi(A)$ are ultimately equal. When $A$ is periodic we shall find that $\varepsilon(A)$ is also periodic and if the projective period is $q$, then $\sigma_{r q-1}(A)=0$ if, and only if, there exists a partial free resolution to dimension $r q-1$ with kernel in the genus of $A$.

In Section 1, we discuss in detail the results needed from the earlier papers in this series, [2] and [3]. The new invariants $\sigma_{n}(A)$ are defined in Section 2 , the stably free resolution theorem is proved in Section 3 and a periodic version is given in Section 4.

There are two appendices. In the first I show that every positive integer does indeed arise as the projective period of a suitable lattice, a result that is very likely known but which I have been unable to find in the literature. The 
second appendix is an afterthought to the decomposition theorem of [2]. It was pointed out to me by Serge Aloneftis that this theorem is valid for all lattices and not just those in the class $\mathscr{Z}$ based on $\mathbb{Z}$. His argument is given in the appendix and is a simple adaptation of the proofs in [2].

It is a pleasure to dedicate this paper to Tim Wall. My first visit to Australia was the result of an invitation by him to the University of Sydney in 1971 . The crucial idea for establishing the existence of the Swan invariants of this paper occurred to me during a brief return visit in 1985 .

\section{Survey of earlier results}

We shall employ here the same notation, conventions and terminology as in [2] and [3], but I repeat in this section sufficient material to save the reader from having to refer to the earlier work (unless, of course, he is interested in proofs).

Fix now a finite group $G$ and consider a $\mathbb{Z} G$-lattice $A$. A projective resolution

$$
\cdots \rightarrow P_{n} \rightarrow P_{n-1} \rightarrow \cdots \rightarrow P_{0} \rightarrow A \rightarrow 0,
$$

where $C_{n}$ is the image of $P_{n}$ in $P_{n-1}$ if $n>0$ and $C_{0}=A$, will be denoted by $(P, C)$ or $P_{*}$. We call $(P, C)$ minimal if $P_{n} \rightarrow C_{n}$ is a minimal projective presentation of $C_{n}$ for all $n \geq 0$ : this means that the kernel $C_{n+1}$ has no non-zero projective module as a direct summand.

Comparing projective resolutions involves the notion of genus. Recall that $\mathbb{Z} G$-lattices $A$ and $B$ belong to the same genus (are locally isomorphic) if and only if $A_{(G)} \simeq B_{(G)}$, where $A_{(G)}=A \otimes_{\mathbb{Z}} \mathbb{Z}_{(G)}$ and $\mathbb{Z}_{(G)}$ is the subring of $\mathbb{Q}$ consisting of all rational numbers $a / b$ with $b$ prime to $|G|$.

An important type of decomposition of a lattice that is unique to within genus is a projective excision. This is a decomposition $A=A^{\prime} \oplus P$, where $P$ is $\mathbb{Z} G$-projective and $A^{\prime}$ has no non-zero projective direct summand. Then $A^{\prime}$ is called an $A$-core and $d_{G}\left(P_{(G)}\right)$ (the minimum number of module generators of $\left.P_{(G)}\right)$ is called the projective rank $\operatorname{pr} A$ of $A$. If $\operatorname{pr} A=0$ we shall say $A$ is core-equal. So all the kernels of a minimal projective resolution of $A$ are core-equal.

If $P_{*}$ and $P_{*}^{\prime}$ are projective resolutions of $A$ with the property that $\operatorname{rank} P_{n}=\operatorname{rank} P_{n}^{\prime}$ for all $n \geq 0$, then $P_{*}$ and $P_{*}^{\prime}$ belong to the same genus: this means that $\left(P_{*}\right)_{(G)}$ and $\left(P_{*}^{\prime}\right)_{(G)}$ are isomorphic augmented complexes over $A_{(G)}$. Two minimal projective resolutions always have the same rank sequences and hence they belong to the same genus. Moreover, an arbitrary 
projective resolution $(P, C)$ of $A$ factors as

$$
(P, C) \simeq\left(P^{\prime}, C^{\prime}\right) \oplus(Q, D),
$$

where $\left(P^{\prime}, C^{\prime}\right)$ is a minimal projective resolution of $A$ and $(Q, D)$ is a projective resolution of 0 . Thus, $C_{n} \simeq C_{n}^{\prime} \oplus D_{n}$ is a projective excision of $C_{n}$ for all $n \geq 1$. We propose to call the above decomposition a projective excision of the complex $(P, C)$. All the non-standard results mentioned so far are proved in [2, Section 3].

Now let $(E, K)$ be a free resolution of $A$. It is minimal if $E_{n} \rightarrow K_{n}$ is a minimal free presentation of $K_{n}$ for all $n \geq 0$ : this means that $d_{G}\left(E_{n}\right)=$ $d_{G}\left(K_{n}\right)$. The rank sequences of two minimal free resolutions may differ, but only if $\mathbb{Z} G$ fails to allow cancellation. When $\mathbb{Z} G$ does allow cancellation, then all minimal free resolutions belong to the same genus. we need later the fact that a minimal free presentation $0 \rightarrow K \rightarrow E \rightarrow L \rightarrow 0$ has $K$ coreequal if, and only if, $L$ is a Swan module; otherwise $\operatorname{pr} K=1$. Recall that a Swan module is a lattice $L$ satisfying $d_{G}(L)=d_{G}\left(L_{(G)}\right)$. These results are all proved in [3, Section 2].

Rank sequences may be compared by partial Euler characteristics. If $P_{*}$ is a projective resolution of $A$ then

$$
\chi_{n}(P)=\sum_{i=0}^{n}(-1)^{n-i} \operatorname{rank} P_{i}
$$

and the infimum of the set $\left\{\chi_{n}(P) \mid\right.$ all $\left.P_{*}\right\}$ is $\chi_{n}(A)$, the $n$th partial projective Euler characteristic of $A$. The resolution $P_{*}$ is minimal if and only if $P_{*}$ realises $\chi(A): \chi_{n}(P)=\chi_{n}(A)$ for all $n \geq 0[2,(3.4)]$. If $E_{*}$ is a free resolution of $A$ we define

$$
\varepsilon_{n}(E)=\sum_{i=0}^{n}(-1)^{n-i} d_{G}\left(E_{i}\right)
$$

and set $\varepsilon_{n}(A)=\inf \left\{\varepsilon_{n}(E) \mid\right.$ all $\left.E_{*}\right\}$. This is called the $n t h$ partial free Euler characteristic of $A$. One should note that $|G| \varepsilon_{n}(E)=\chi_{n}(E)$ and (since projective $\mathbb{Z}_{(G)} G$-modules are free) $\chi_{n}(P)=|G| \varepsilon_{n}\left(P_{(G)}\right)$. Moreover by [2, (3.4)], $\chi_{n}(A)=|G| \varepsilon_{n}\left(A_{(G)}\right)$. (The functions $\chi$ and $\varepsilon$ are defined over $\mathbb{Z}_{(G)}$ just as they are over $\mathbb{Z}$.) Because we are here interested in comparing the two types of Euler characteristic we shall work with $\varepsilon\left(-{ }_{(G)}\right)$ rather than with $\chi(-)$.

If $(P, C)$ is a projective resolution and $s>r \geq 0$, we denote by ${ }_{s}(P, C)_{r}$ the segment $[s, r]$ of $(P, C)$ :

$$
0 \rightarrow C_{s+1} \rightarrow P_{s} \rightarrow \cdots \rightarrow P_{r} \rightarrow C_{r} \rightarrow 0 .
$$


Then ${ }_{n}(P, C)_{0}$ is the partial (or truncated) resolution to dimension $n$. Note that we choose to write $[s, r]$ rather than $[r, s]$; this is done to conform with the convention that suffices increase to the left in a resolution.

It is clear that $\varepsilon_{n}\left(P_{(G)}\right)$ depends only on ${ }_{n}(P, C)_{0}$. We use our Euler characteristic notation also for partial resolutions. Note that a projective excision of $(P, C)$ determines a projective excision of each truncation. If the projective excision is $(P, C) \simeq\left(P^{\prime}, C^{\prime}\right) \oplus(Q, D)$, then we easily verify

$$
\varepsilon_{n}\left(P_{(G)}\right)=\varepsilon_{n}\left(P_{(G)}^{\prime}\right)+\operatorname{pr} D_{n+1} .
$$

Periodicity is usually defined in terms of the behaviour of the functor $\operatorname{Ext}_{\mathrm{z} G}^{*}(A$,$) . An equivalent, and for our purposes more useful, definition is$ this: the lattice $A$ has projective period $q$ if $q$ is the smallest positive integer so that there exists a minimal projective resolution $(P, C)$ of an $A$-core $A^{\prime}$ in which $C_{r q} \simeq A^{\prime}$ for all $r \geq 1$ and each segment $[r q-1,(r-1) q]$ is a repeat of $[q-1,0]$. (Cf. $[3,(3.1)]$.)

All the results noted in this section will be used freely and usually without further reference in the rest of this paper.

\section{The Swan invariants of a lattice}

We shall need to work with the projective class group of $\mathbb{Z} G$. Let $K_{0}(\mathbb{Z} G)$ be the Grothendieck group of projective $\mathbb{Z} G$-modules and $\{P\}$ denote the element corresponding to the projective module $P$. The homomorphism $\delta: \mathbb{Z} \rightarrow K_{0}(\mathbb{Z} G)$ given by $\delta(n)=n\{\mathbb{Z} G\}$ is split by a homomorphism $\delta^{\prime}: K_{0}(\mathbb{Z} G) \rightarrow \mathbb{Z}$, where $\delta^{\prime}\{P\}=d_{G}(\mathbb{Q} P)$. Both $\operatorname{Ker} \delta^{\prime}$ and Coker $\delta$ have been called the projective class group of $\mathbb{Z} G$. Four our purposes it is more convenient to work with Coker $\delta$, which we therefore call here the projective class group of $\mathbb{Z} G$. We write $K(\mathbb{Z} G)=\operatorname{Coker} \delta$ and $[P]$ for the element determined $\{P\}$. Thus $[E]=0$ if $E$ is $\mathbb{Z} G$-free and $[P]=[Q]$ if and only if $P \oplus E \simeq Q \oplus F$ for suitable free modules $E$ and $F$. The projective module $P$ is called stably free if $[P]=0$ and thus, whenever $\mathbb{Z} G$ allows cancellation, stably free implies free. If $P_{*}$ is a projective resolution of $A$ we put

$$
\varepsilon_{n}[P]=\sum_{i=0}^{n}(-1)^{n-i}\left[P_{i}\right] .
$$

Let $M$ be a finite $\mathbb{Z} G$-module of order prime to $|G|$ and let

$$
0 \rightarrow Q \rightarrow P \rightarrow M \rightarrow 0
$$

be a $\mathbb{Z} G$-projective presentation. Then $Q_{(G)} \simeq P_{(G)}$, whence $Q$ is also projective and $[P]-[Q]$ is an element of $K(\mathbb{Z} G)$ which depends only on $M$ and not 
on the projective resolution (by Schanuel's Lemma). We set $[M]=[P]-[Q]$.

Now let $L$ be a $\mathbb{Z} G$-lattice, write $V=\mathbb{Q} L$ and define $C_{V}$ to be the subgroup of $K(\mathbb{Z} G)$ generated by all $\left[L^{\prime} / L\right]$, where $L^{\prime}$ is a lattice such that $L \subset L^{\prime} \subset V$ and $\left|L^{\prime}: L\right|$ is finite of order prime to $|G|$. (This construction is due to Swan [5, page 198]. An equivalent definition of $C_{V}$ is the subgroup generated by all $[S]$, where $S$ is a simple image of $L$ of order prime to $|G|$.) We remark that $C_{\mathbb{Q}}$ is the much studied subgroup, frequently denoted by $T(\mathbb{Z} G)$, generated by all $[r \mathbb{Z} G+\nu \mathbb{Z}]$, where $r$ is an integer prime to $|G|$ and $\nu=\sum_{g \in G} g$.

Let $(P, C)$ be a minimal resolution of $A$. We know that $(P, C)$ is uniquely determined to within genus and hence the $\mathbb{Q} G$-modules $\mathbb{Q} C_{n}$, $n \geq 0$, are uniquely determined by $A$. Therefore so are the subgroups $S_{n}(A)=C_{\mathrm{Q} C_{n}}$. As $P_{*}$ varies, the elements $\varepsilon_{n}[P]$ may change but our main result here shows that no change occurs modulo $S_{n+1}(A)$.

(2.1) Theorem. If $P_{*}$ and $Q_{*}$ are minimal projective resolutions of $A$ then for each $n \geq 0$,

$$
\varepsilon_{n}[P]-\varepsilon_{n}[Q] \in S_{n+1}(A) .
$$

We give two quite different proofs of this theorem. The second one was shown to me by Peter Linnell and I am grateful to him for permission to include it here. It is representation theoretic and the central idea is to show that if $L$ and $M$ are lattices in the same genus and $L \oplus P=M \oplus Q$ with $P$ and $Q$ projective, then $[P]-[Q] \in C_{\mathbb{Q} L}$. My own proof is more homological and depends on describing globally the relation between two resolutions that belong to the same genus.

We begin with this homological proof of (2.1).

(2.2) Lemma. Let there be given a diagram of $\mathbb{Z} G$-lattices

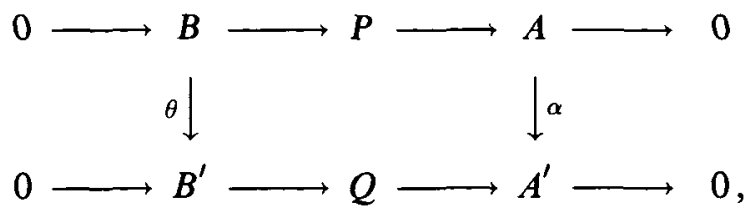

with exact rows, $P$ and $Q$ projective and both $\alpha_{(G)}$ and $\theta_{(G)}$ isomorphisms. Then there exists a commutative diagram

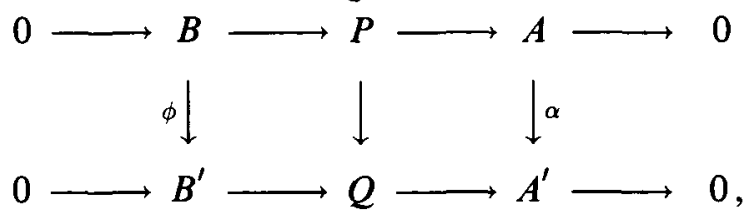

where $\phi_{(G)}$ is an isomorphism. 
Proof. (The reader may like to compare what follows with the proof of (3.5) in [2].) Let $\xi$ be the element in $\operatorname{Ext}_{\mathrm{ZG}}(A, B)$ determined by the top sequence and let $\xi^{\prime}$ in $\operatorname{Ext}_{\mathrm{zG}}\left(A^{\prime}, B^{\prime}\right)$ correspond to the lower one. Now

$$
\operatorname{Ext}_{\mathbf{z G}}(A, B) \otimes \mathbb{Z}_{(G)} \simeq \operatorname{Ext}_{\mathbf{z}_{(G)} G}\left(A_{(G)}, B_{(G)}\right)
$$

and the isomorphisms $\alpha_{(G)}$ and $\theta_{(G)}$ determine an isomorphism

$$
\operatorname{Ext}_{\mathbf{z}_{(G)} G}\left(A_{(G)}, B_{(G)}\right) \stackrel{\sim}{\rightarrow} \operatorname{Ext}_{\mathbf{z}_{(G)} G}\left(A_{(G)}^{\prime}, B_{(G)}^{\prime}\right),
$$

under which $\xi_{(G)} \rightarrow \alpha_{(G)}^{-1} \xi_{(G)} \theta_{(G)}$.

Let $E=\operatorname{End}_{\mathbf{Z} G}\left(B^{\prime}\right)$, so that $E_{(G)} \simeq \operatorname{End}_{\mathbb{Z}_{(G)} G}\left(B_{(G)}^{\prime}\right)$. Since $\xi_{(G)}^{\prime}$ generates $\operatorname{Ext}_{\mathbf{z}_{(G)} G}\left(A_{(G)}^{\prime}, B_{(G)}^{\prime}\right)$ as a right $E_{(G)}$-module [2,(3.6)(iii)], we can find an element $\mu$ in $E_{(G)}$ such that

$$
\alpha_{(G)}^{-1} \xi_{(G)} \theta_{(G)}=\xi_{(G)}^{\prime} \mu .
$$

But $\xi_{(G)}$ generates its Ext-group and hence we can find $\nu \in E_{(G)}$ such that

$$
\xi_{(G)}^{\prime}=\alpha_{(G)}^{-1} \xi_{(G)} \theta_{(G)} \nu
$$

Thus $\xi_{(G)}^{\prime}=\xi_{(G)}^{\prime} \mu \nu$. If $I$ is the annihilator of $\xi_{(G)}^{\prime}$ in $E_{(G)}$, then $\mu \nu-1 \in I$ and so $E_{(G)}=\mu E_{(G)}+I$, whence [1, page 87] there exists an invertible element $\beta$ in $E_{(G)}$ of the form $\beta=\mu+\delta$, with $\delta \in I$. Let $\beta^{-1}=\rho \otimes \frac{1}{r}$, where $\rho$ is an injective endomorphism of $E$ and $r$ is prime to $|G|$. We can and shall assume $r \equiv 1(\bmod |G|)$. Then

$$
\begin{aligned}
(\xi \theta \rho)_{(G)} & =\xi_{(G)} \theta_{(G)} \rho_{(G)}=\xi_{(G)} \theta_{(G)} \beta^{-1} r 1_{B_{(G)}^{\prime}} \\
& =\alpha_{(G)} \xi_{(G)}^{\prime} \beta \beta^{-1} r 1_{B_{(G)}^{\prime}}=\left(\alpha \xi^{\prime} r 1_{B^{\prime}}\right)_{(G)}
\end{aligned}
$$

and thus $\xi \theta p=\alpha \xi^{\prime} r 1_{B^{\prime}}$.

We may now construct extensions as follows:

$$
\begin{aligned}
& \xi \quad 0 \rightarrow B \rightarrow P \rightarrow A \rightarrow 0 \\
& \xi \theta \rho \quad 0 \rightarrow \stackrel{\theta \rho \downarrow}{B^{\prime}} \rightarrow \stackrel{\downarrow}{T} \rightarrow \stackrel{\downarrow}{\perp} \rightarrow 0 \text { : the push-out to } \theta \rho \\
& \alpha\left(\xi^{\prime} r 1_{B^{\prime}}\right) \quad 0 \rightarrow B^{\prime} \rightarrow S \rightarrow A \rightarrow 0 \quad \rightarrow \quad \text { the pull-back to } \alpha \\
& \xi^{\prime} r 1_{B^{\prime}} \quad 0 \rightarrow \stackrel{=\downarrow}{B^{\prime}} \rightarrow \stackrel{\downarrow}{R} \rightarrow \stackrel{\downarrow}{A^{\prime}} \rightarrow 0 \text { : the push-out to } r 1_{B^{\prime}} \\
& \xi^{\prime} \quad 0 \rightarrow \begin{array}{l}
r \uparrow \\
B^{\prime}
\end{array} \rightarrow \stackrel{\uparrow}{Q} \rightarrow \stackrel{\uparrow=}{A^{\prime}} \rightarrow 0 .
\end{aligned}
$$


Since $\xi \theta \rho=\alpha \xi^{\prime} r 1_{B^{\prime}}$, the corresponding extensions are equivalent. Also the extensions $(R)$ and $(Q)$ are equivalent because $r \equiv 1(\bmod |G|)$. So we obtain a diagram as in the statement of (2.2).

(2.3) Lemma. Given minimal projective resolutions $(P, C),(Q, D)$ of $A$, there exists a complex homomorphism $\varphi:(P, C) \rightarrow(Q, D)$ over the identity on $A$ so that $\varphi_{(G)}$ is an isomorphism.

Proof. We construct $\varphi$ inductively. So suppose we have already established the existence of a complex homomorphism with the required properties on truncated resolutions,

$$
\varphi(n):{ }_{n}(P, C)_{0} \rightarrow{ }_{n}(Q, D)_{0} .
$$

Then we have

$$
\begin{aligned}
& 0 \rightarrow C_{n+2} \rightarrow P_{n+1} \rightarrow C_{n+1} \rightarrow 0 \\
& \downarrow \varphi(n) \\
& 0 \rightarrow D_{n+2} \rightarrow Q_{n+1} \rightarrow D_{n+1} \rightarrow 0
\end{aligned}
$$

where $C_{n+2}$ and $D_{n+2}$ are in the same genus (by minimality). Hence (2.2) yields a homomorphism $\theta: P_{n+1} \rightarrow Q_{n+1}$ which induces $\varphi(n)$ and is such that $\theta_{(G)}$ is an isomorphism. We define $\varphi(n+1)$ to be $\theta$ on $P_{n+1}$ and $\varphi(n)$ on $P_{i}$ for $i \leq n$. This completes the proof.

The next lemma shows that, in the situation of (2.3), for each $n \geq 0$, $\varepsilon_{n}[P]-\varepsilon_{n}[Q] \in S_{n+1}(A)$ and Theorem 2.1 is proved.

(2.4) Lemma. Let there be given a commutative diagram of $\mathbb{Z} G$-lattices,

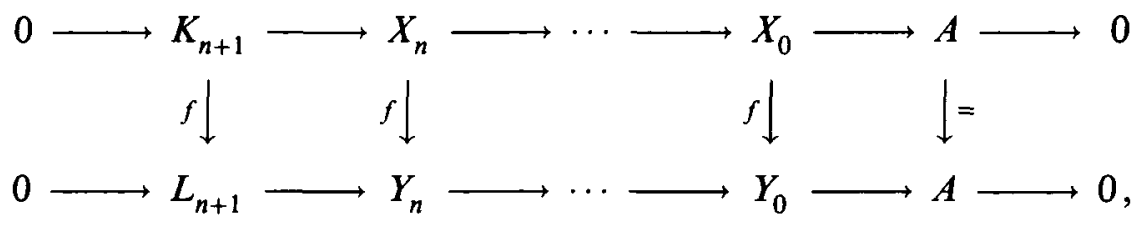

with exact rows, $X_{i}$ and $Y_{i}$ being $\mathbb{Z} G$-projective $(0 \leq i \leq n)$ and $f_{(G)}$ an isomorphism. Then

$$
\left[X_{n}\right]-\left[X_{n-1}\right]+\cdots \equiv\left[Y_{n}\right]-\left[Y_{n-1}\right]+\cdots \quad\left(\bmod C_{V}\right),
$$

where $V=\mathbb{Q} K_{n+1}=\mathbb{Q} L_{n+1}$.

This lemma is a slight generalization of a result of Swan $[5,5.1]$. For the convenience of the reader we repeat Swan's proof.

Proof. Let $M_{*}$ be the mapping cone for $f: X_{*} \rightarrow Y_{*}$. Thus $M_{i}=$ $X_{i-1} \oplus Y_{i}, 0 \leq i \leq n+1$ (where $X_{-1}=Y_{n+1}=0$ ), and we have an exact 
sequence

$$
0 \rightarrow H_{n+1}(M) \rightarrow H_{n}(X) \rightarrow H_{n}(Y) \rightarrow H_{n}(M) \rightarrow \cdots \rightarrow H_{0}(Y) \rightarrow H_{0}(M) \rightarrow 0 .
$$

Here $f$ induces $H_{*}(X) \rightarrow H_{*}(Y)$. Since $X_{*}$ and $Y_{*}$ are partial projective resolutions, $H_{i}(X)=H_{i}(Y)=0$ for $0<i<n, H_{0}(X) \rightarrow H_{0}(Y)$ is an isomorphism and

$$
H_{n}(X)=K_{n+1} \rightarrow L_{n+1}=H_{n}(Y)
$$

is injective with cokernel of order prime to $|G|$. Consequently $H_{i}(M)=0$ for $0 \leq i \leq n-1$ and $i=n+1$, while $\left|H_{n}(M)\right|$ is prime to $|G|$.

Now consider the projective complex

$$
0 \rightarrow M_{n+1} \rightarrow \cdots \rightarrow M_{0} \rightarrow 0
$$

and let $M_{i+1}^{\prime}$ be the kernel of $M_{i} \rightarrow M_{i-1}(1 \leq i \leq n)$. Then

$$
0 \rightarrow M_{n+1} \rightarrow M_{n+1}^{\prime} \rightarrow H_{n}(M) \rightarrow 0
$$

is exact and so $M_{n+1}^{\prime}$ is projective because $M_{n+1}$ is projective. The projectivity of $M_{*}$ and the exactness of $M_{*}$ at all dimensions at most $n-1$ gives splittings

$$
M_{i} \simeq M_{i}^{\prime} \oplus M_{i+1}^{\prime} \quad(1 \leq i \leq n)
$$

and $M_{1}^{\prime}=M_{0}$. We have therefore

$$
\left[M_{n+1}\right]-\left[M_{n}\right]+\cdots+(-1)^{n+1}\left[M_{0}\right]=\left[M_{n+1}\right]-\left[M_{n+1}^{\prime}\right]=-\left[H_{n}(M)\right] .
$$

But $\left[M_{n+1}\right]-\left[M_{n}\right]+\cdots=\left(\left[X_{n}\right]-\left[X_{n-1}\right]+\cdots\right)-\left(\left[Y_{n}\right]-\left[Y_{n-1}\right]+\cdots\right)$ and $\left[H_{n}(M)\right]=\left[L_{n+1}\right]-\left[K_{n+1}\right] \in C_{V}$. This completes the proof.

We now turn to Linnell's proof of (2.1).

(2.5) Lemma. Let $I$ be the annihilator in $\mathbb{Z} G$ of the $\mathbb{Z} G$-lattice $L$ and $J$ the annihilator in $\mathbb{Z} G$ of $I$ as right ideal. Then

(i) $I$ and $J$ are two sided ideals and both $\mathbb{Z} G / I$ and $\mathbb{Z} G / J$ are lattices;

(ii) $\mathbb{Q} I \oplus \mathbb{Q} J=\mathbb{Q} G$ and $|G| \in I+J$;

(iii) $C_{\mathbb{Q} L}=C_{Q J}$

Proof. (i) is obvious. Turning to (ii), since $\mathbb{Q} G$ is semi-simple and $\mathbb{Q} I$ is a two sided ideal, $\mathbb{Q} G=\mathbb{Q} I \oplus W$, where $W$ is the annihilator of $\mathbb{Q} I$. Hence $W=\mathbb{Q} J$. Let $\Lambda$ be a maximal order containing $\mathbb{Z} G$ and define $I_{0}=\mathbb{Q} I \cap \Lambda$, $J_{0}=\mathbb{Q} J \cap \Lambda$. Then $\Lambda=I_{0} \oplus J_{0}$ and therefore $|G| \in|G| I_{0}+|G| J_{0} \subseteq I+J$. Thus (ii) is established. 
Finally, if $e$ is a primitive central idempotent then $(\mathbb{Q} L) e \neq 0$ if, and only if, $e \notin \mathbb{Q} I$ which is equivalent to $e \in \mathbb{Q} J$. Hence $\mathbb{Q} L$ and $\mathbb{Q} J$ involve the same simple $\mathbb{Q} G$-modules. Now if a simple $\mathbb{Q} G$-module $W$ is a summand of $\mathbb{Q} L$ and $D$ is a $\mathbb{Z}$-form of $W$ (meaning that $D$ is a $\mathbb{Z} G$-lattice contained in $W$ such that $\mathbb{Q} D=W$ ), then every finite simple image of $D$ of characteristic prime to $|G|$ belongs to $C_{\mathbb{Q} L}$. Moreover $C_{\mathbb{Q} L}$ is generated by these finite simple modules as $W$ varies in $\mathbb{Q} L$. We conclude that $C_{\mathbb{Q} L}=C_{\mathbb{Q} J}$.

(2.6) Lemma. Let $L$ and $M$ be $\mathbb{Z} G$-lattices in the same genus and suppose $L \oplus P \simeq M \oplus Q$, where $P$ and $Q$ are projective. Then there exists an injective $\mathbb{Z} G$-homomorphism $\theta: P \rightarrow Q$ so that $[Q / P \theta] \in C_{\mathbb{Q} L}$.

Proof. By (2.5)(i), $I \cap J=0$ and thus the commutative diagram

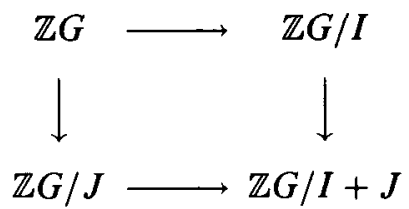

is a fibre product diagram. It follows that the same is true of

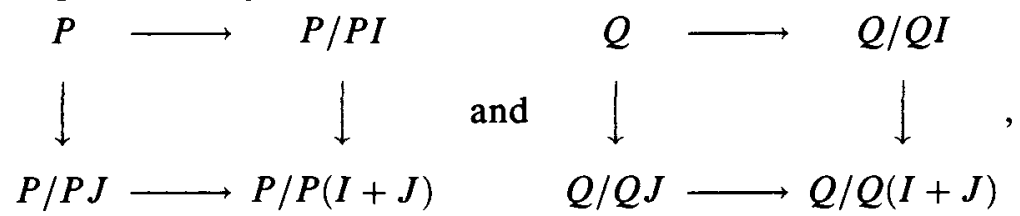

because $P I \cap P J=0$ as $P$ is a direct summand of some $\mathbb{Z} G^{(m)}$; similarly for $Q$. Now $L / L J=L / L(I+J)$ and the right hand side is finite by (2.5) (ii); also $M / M J \simeq L / L J$ since $L$ and $M$ lie in the same genus. The isomorphism

$$
L / L J \oplus P / P J \simeq M / M J \oplus Q / Q J
$$

induces an isomorphism $n(P / P J) \simeq n(Q / Q J)$, where $n=|L: L J|$ and this gives an isomorphism $\alpha: P / P J \simeq Q / Q J$ (because these modules are lattices by $(2.5)(i))$. We also have an isomorphism

$$
L / L I \oplus P / P I \simeq M / M I \oplus Q / Q I .
$$

By semi-local cancellation we obtain an injective homomorphism $\beta: P / P I \rightarrow$ $Q / Q I$, whose cokernel is finite of order prime to $|G|$.

We now have homomorphisms

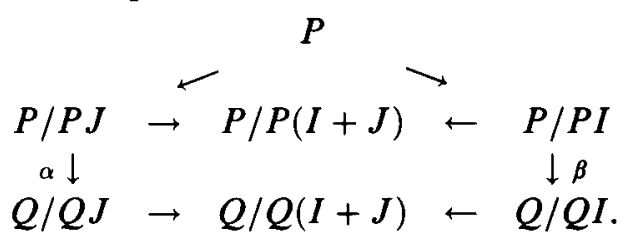


Let $\bar{\alpha}$ be the homomorphism $P / P(I+J) \rightarrow Q / Q(I+J)$ induced by $\alpha$ and $\bar{\beta}$ that induced by $\beta$. We claim that $\beta$ can be chosen so that $\bar{\beta}=\bar{\alpha}$. Since $\mid$ Coker $\beta \mid$ prime to $|G|, \beta$ induces an isomorphism modulo $|G|$ and then, because $|G| \in I+J, \bar{\beta}$ is an isomorphism. We may lift the automorphism $\bar{\alpha} \bar{\beta}^{-1}$ of $P / P(I+J)$ to an endomorphism $\rho$ of $P /(P I+|G| P)$, since this module is projective over $\mathbb{Z} G /(|G| \mathbb{Z} G+I)$. Thus $\rho$ maps to a unit in $\operatorname{End}_{\mathrm{Z} G}(P / P(I+J))$ and so, because $\operatorname{End}_{\mathrm{Z} G}(P /(P I+|G| P))$ is Artinian, we can find a unit $\sigma$ in $\operatorname{End}_{\mathbb{Z} G}(P /(P I+|G| P))$ having the same image as $\rho$ (e.g. $[1$, page 87$])$. Because $P / P I$ is projective over $\mathbb{Z} G / I$, we may lift $\sigma$ to an endomorphism $\gamma$ of $P / P I$ and $\gamma$ has finite cokernel of order prime to $|G|$ since $\gamma$ induces the automorphism $\sigma$. It follows that $\mid$ Coker $\gamma \beta \mid$ is prime to $|G|$ and $\bar{\gamma} \bar{\beta}=\bar{\alpha}$. So we may now replace $\beta$ by $\gamma \beta$ and we shall call this new map $\beta$.

Now $\bar{\alpha}=\bar{\beta}$ implies by (1) that the two homomorphisms

$$
\begin{aligned}
& P \rightarrow P / P J \rightarrow Q / Q J \rightarrow Q / Q(I+J), \\
& P \rightarrow P / P I \rightarrow Q / Q I \rightarrow Q / Q(I+J)
\end{aligned}
$$

are the same, whence the fibre product property yields a homomorphism $\theta: P \rightarrow Q$. As $\theta$ induces $\alpha$ and $\beta$, both of which are injective, so $x \theta=0$ implies $x \in P J \cap P I=0$, showing $\theta$ is injective. But $\alpha$ is also surjective, whence $Q=P \theta+Q J$ and the cokernel of $\beta$ is $Q /(P \theta+Q I)=Q / P \theta$ because $Q I=(P \theta+Q J) I=P \theta I$. We know $\beta$ has finite cokernel of order prime to $|G|$; consequently $[Q / P \theta]$ exists and belongs to $C_{\mathrm{Q} R}$, where $R=Q / Q I$. Clearly $C_{\mathbb{Q} R}=C_{\mathbb{Q} G / \mathbb{Q} I}$ and $\mathbb{Q} G / \mathbb{Q} I=\mathbb{Q} J$. Now (2.5)(iii) completes the proof of the lemma.

Theorem 2.1 is an immediate consequence of $(2.6)$ if we recall that $[Q / P \theta]$ $=[Q]-[P]$. If $(P, C),(Q, D)$ are minimal projective resolutions of $A$, then by Schanuel's lemma,

$$
C_{n+1} \oplus Q_{n} \oplus P_{n-1} \oplus \cdots \simeq D_{n+1} \oplus P_{n} \oplus Q_{n-1} \oplus \cdots
$$

and so by (2.6),

$$
\left[P_{n} \oplus Q_{n-1} \oplus \cdots\right]-\left[Q_{n} \oplus P_{n-1} \oplus \cdots\right] \in C_{Q C_{n+1}},
$$

as required.

If $(P, C)$ is a minimal projective resolution of $A$, we now know that $\varepsilon_{n}[P]$ determines an element of $K(\mathbb{Z} G) / S_{n+1}(A)$, independent of the choice of $P_{*}$. We shall write

$$
\sigma_{n}(A)=\varepsilon_{n}[P]+S_{n+1}(A)
$$

and call this the $n$th Swan invariant of $A$. 
Suppose $A$ is non-periodic. Then for all sufficiently large $n, \mathbb{Q} G$ is a direct summand of $\mathbb{Q} C_{n+1}$ (cf. the proof of (3.5) in [3], which implies $S_{n+1}(A)=K(\mathbb{Z} G)$. Hence $\sigma_{n}(A)$ is ultimately zero and we have established

(2.7) Proposition. If $A$ is a non-periodic $\mathbb{Z} G$-lattice, there exists an integer $N \geq 0$ such that $\sigma_{n}(A)=0$ for all $n \geq N$.

The behaviour of the Swan invariant sequence for a periodic lattice will be discussed in Section 4 .

\section{Stably free resolutions}

Our first result connects the Swan invariants with the two partial Euler characteristics.

(3.1) Theorem. If $A$ is a $\mathbb{Z} G$-lattice, then for all $n \geq 0, \sigma_{n}(A)=0$ if and only if $\varepsilon_{n}(A)=\varepsilon_{n}\left(A_{(G)}\right)$.

We begin the proof with an elementary extension of Swan's inequality $d_{G}(A) \leq d_{G}\left(A_{(G)}\right)+1$.

(3.2) Proposition.

(i) If the free resolution $(E, K)$ of $A$ realises $\varepsilon_{n}(A)$, then $(E, K)$ is minimal in dimension $n$.

(ii) If the free resolution $(E, K)$ of $A$ is minimal in dimension $n$ then $\varepsilon_{n}(E)$ equals $\varepsilon_{n}\left(A_{(G)}\right)$ or $\varepsilon_{n}\left(A_{(G)}\right)+1$ according as $K_{n}$ is a Swan module and $K_{n+1}$ is core-equal or $K_{n}$ is not a Swan module and $\operatorname{pr} K_{n+1}=1$.

(iii) $\varepsilon_{n}(A)_{(G)} \leq \varepsilon_{n}(A) \leq \varepsilon_{n}\left(A_{(G)}\right)+1$.

Proof. (i) If we continue the segment $[n-1,0]$ of $E_{*}$ with a minimal free presentation of $K_{n}$ we obtain a new free segment [ $\left.n, 0\right]$ and hence

$$
\varepsilon_{n}(E) \geq d_{G}\left(K_{n}\right)-d_{G}\left(E_{n-1}\right)+\cdots \geq \varepsilon_{n}(A) .
$$

By hypothesis $\varepsilon_{n}(E)=\varepsilon_{n}(A)$ whence (i) follows.

(ii) Take a projective excision

$$
(E, K) \simeq(P, C) \oplus(Q, D),
$$

where $(P, C)$ is a minimal projective resolution of $A$ and $(Q, D)$ is a projective resolution of 0 . Then $K_{n+1} \simeq C_{n+1} \oplus D_{n+1}$, whence

$$
\varepsilon_{n}(E)=\varepsilon_{n}\left(P_{(G)}\right)+\operatorname{pr} K_{n+1}=\varepsilon_{n}\left(A_{(G)}\right)+\operatorname{pr} K_{n+1} \text {. }
$$


Since $E_{n} \rightarrow K_{n}$ is minimal free, $\operatorname{pr} K_{n+1}$ is 0 or 1 according as $K_{n}$ is, or is not, a Swan module [3, (2.2)].

(iii) The first inequality is immediate from the meaning of $\varepsilon_{n}$ and the second follows from parts (i) and (ii).

Assume $(P, C)$ is a projective resolution of $A$ that is stably free at some dimension $k>0$. We define an adjustment of $(P, C)$ at dimension $k$ to be a new projective resolution $\left(P^{\prime}, C^{\prime}\right)$ which coincides with $(P, C)$ on $[k-2,0]$ and $(\infty, k+1]$, while $P_{k}^{\prime}=P_{k} \oplus F$ and $P_{k-1}^{\prime}=P_{k-1} \oplus F$, where $F$ is $\mathbb{Z} G$-free and is chosen so as to make $P_{k}^{\prime}$ free. Thus $\left(P^{\prime}, C^{\prime}\right)$ is

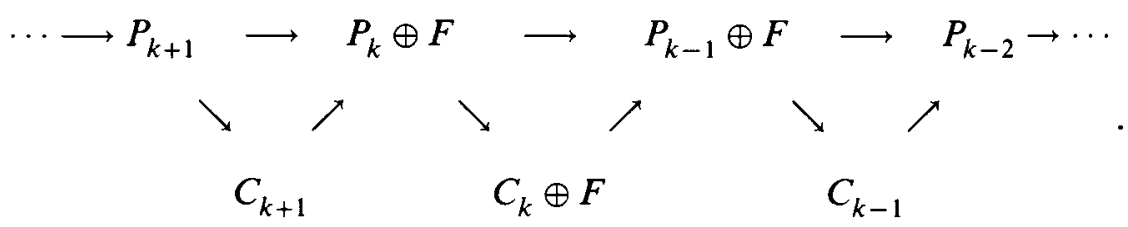

It is important to note that an adjustment at $k$ does not affect $\varepsilon_{n}\left(P_{(G)}\right)$ or $\varepsilon_{n}[P]$ for any $n \geq k$.

(3.3) Lemma. Let $(E, K)$ be a stably free partial resolution of $A$ to dimension $n-1$ with $E_{0}$ free. If $\sigma_{n}(A)=0$ then $(E, K)$ can be extended to dimension $n$ by a stably free and minimal projective presentation $E_{n} \rightarrow K_{n}$ to give $\varepsilon_{n}\left(E_{(G)}\right)=\varepsilon_{n}\left(A_{(G)}\right)=\varepsilon_{n}(A)$. The presentation $E_{n} \rightarrow K_{n}$ can be chosen to be minimal free if $K_{n}$ is a Swan module.

Proof. Extend $(E, K)$ to dimension $n$ by a minimal projective presentation $0 \rightarrow L_{n+1} \rightarrow R_{n} \rightarrow K_{n} \rightarrow 0$ and call the extension $(R, L)$. If

$$
(R, L) \simeq(P, C) \oplus(Q, D)
$$

is a projective excision, then $L_{n+1}$ being core-equal implies $D_{n+1}=0$ and so $\varepsilon_{n}[Q]=0$. Hence $\varepsilon_{n}[R]=\varepsilon_{n}[P]$ whence $\varepsilon_{n}[R] \in S_{n+1}(A)$ since $\sigma_{n}(A)=0$ (Theorem 2.1). But

$$
\varepsilon_{n}[R]=\left[R_{n}\right]-\left[E_{n-1}\right]+\cdots=\left[R_{n}\right]
$$

and so $\left[R_{n}\right] \in S_{n+1}(A)$. We may now apply Lemma 4.2 in [5] to obtain a minimal projective presentation $0 \rightarrow K_{n+1} \rightarrow E_{n} \rightarrow K_{n} \rightarrow 0$ with $\left[E_{n}\right]=0$. If $(E, K)$ denotes the original $(E, K)$ extended by this stably free term, then

$$
\begin{aligned}
\varepsilon_{n}\left(E_{(G)}\right) & =\varepsilon_{n}\left(R_{(G)}\right) \\
& =\varepsilon_{n}\left(P_{(G)}\right) \quad\left(\text { since } D_{n+1}=0\right) \\
& =\varepsilon_{n}\left(A_{(G)}\right) .
\end{aligned}
$$


Now adjust $E_{*}$ at all positive dimensions to obtain a free partial resolution $E_{*}^{\prime}$. Then $\varepsilon_{n}\left(E_{(G)}\right)=\varepsilon_{n}\left(E^{\prime}\right)$ and, of course, $\varepsilon_{n}\left(E^{\prime}\right) \geq \varepsilon_{n}(A)$, whence $\varepsilon_{n}(A)=$ $\varepsilon_{n}\left(E_{(G)}\right)$, as required. Finally, note that if $K_{n}$ is a Swan module we may choose $R_{n}$ to be free.

Proof of Theorem 3.1. Lemma 3.3 establishes the "only if" half. So now assume $\varepsilon_{n}(A)=\varepsilon_{n}\left(A_{(G)}\right)$ and choose a free resolution $(E, K)$ realising $\varepsilon_{n}(A)$. Then $E_{n} \rightarrow K_{n}$ is a minimal free presentation and $K_{n+1}$ is core-equal (Proposition (3.2)). Hence if $(P, C)$ is a minimal projective summand of $(E, K)$ in a projective excision, $\varepsilon_{n}[E]=\varepsilon_{n}[P]$ and the left hand side is 0 , whence $\sigma_{n}(A)=0$. This completes the proof of Theorem 3.1.

For any $\mathbb{Z} G$-lattice $A$ we define

$$
I(A)=\left\{n \in \mathbb{Z} \mid \sigma_{n}(A)=0\right\} .
$$

(3.4) THEOREM. Given a $\mathbb{Z} G$-lattice $A$, there exists a projective resolution $(E, K)$ of $A$ with the following properties:

(i) $E_{0} \rightarrow A$ is a minimal free presentation;

(ii) if $n \in I(A), E_{n} \rightarrow K_{n}$ is minimal projective and stably free;

(iii) if $n \notin I(A), E_{n} \rightarrow K_{n}$ is minimal free and not minimal projective;

(iv) $\varepsilon_{n}\left(E_{(G)}\right)=\varepsilon_{n}(A)$ for all $n \geq 0$.

\section{REMARKS.}

(1) Conditions (ii) and (iii) for $n=0$ do not conflict with (i) because $0 \in I(A)$ if and only if $A$ is a Swan module, in which case $E_{0} \rightarrow A$ can be chosen minimal free and minimal projective. Moreover, for any $n$, if $n \in I(A)$ and $K_{n}$ is a Swan module, then $E_{*}$ can be taken free and minimal projective at $n$ (Lemma 3.3).

(2) If $\mathbb{Z} G$ allows cancellation, then stably free means free and hence $E_{*}$ is a minimal free resolution which, by (iv), realises $\varepsilon(A)$. Then every minimal free resolution realises $\varepsilon(A)$ because any two such have the same rank sequences [3, (2.5)]. We can see this without using Swan invariants as follows. Suppose $(E, K)$ and and $\left(E^{\prime}, K^{\prime}\right)$ are free and minimal at dimension $n$ and that $(E, K)$ realises $\varepsilon_{n}(A)$. We must show that $\left(E^{\prime}, K^{\prime}\right)$ also realises $\varepsilon_{n}(A)$. Now $K_{n} \oplus$ free $\simeq K_{n}^{\prime} \oplus$ free, whence $K_{n}$ is a Swan module if and only if $K_{n}^{\prime}$ is one [3, (2.3)]. Hence $\varepsilon_{n}(E)=\varepsilon_{n}\left(E^{\prime}\right)$ by (3.2)(ii).

(3) Two resolutions with the properties of (3.4) must have the same rank sequences and so they belong to the same genus.

(4) If we adjust $E_{*}$ at all integers in $[n, 1] \cap I(A)$, we obtain a partial free resolution to dimension $n$ that realises $\varepsilon_{n}(A)$. 
Proof of (3.4). Assume $(E, K)$ has been constructed up to dimension $n-1$. If $n \in I(A)$, then (3.3) gives what we want. Suppose $n \notin I(A)$. Choose a minimal free presentation $E_{n} \rightarrow K_{n}$ and adjust $(E, K)$ at all dimensions in $[n-1,1] \cap I(A)$, so producing a partial free resolution ${ }_{n-1}\left(E^{\prime}\right)_{0}$ with $\varepsilon_{n-1}\left(E^{\prime}\right)=\varepsilon_{n-1}\left(E_{(G)}\right)$. Now $\varepsilon_{n}\left(E_{(G)}\right)=d_{G}\left(E_{n}\right)-\varepsilon_{n-1}\left(E^{\prime}\right)$ and by (3.2), $K_{n}$ is not a Swan module: otherwise $\varepsilon_{n}\left(E_{n} \rightarrow E_{n-1}^{\prime} \rightarrow \cdots\right)=\varepsilon_{n}\left(E_{(G)}\right)=$ $\varepsilon_{n}\left(A_{(G)}\right)$. Hence $\varepsilon_{n}\left(E_{(G)}\right)=\varepsilon_{n}\left(A_{(G)}\right)+1=\varepsilon_{n}(A)$ and $E_{n} \rightarrow K_{n}$ is not minimal projective. This completes the proof.

(3.5) Corollary. Let $A$ be a periodic $\mathbb{Z} G$-lattice. If $s$ is a multiple of the projective period of $A$, then $\sigma_{s-1}(A)=0$ is a necessary and sufficient condition for the existence of a partial free resolution

$$
0 \rightarrow B \rightarrow F_{s-1} \rightarrow \cdots \rightarrow F_{0} \rightarrow A \rightarrow 0
$$

where $B$ is in the genus of $A$ and admits a projective excision of the form $B=B^{\prime} \oplus \mathbb{Z} G^{(m)}$.

Proof. Suppose $\sigma_{s-1}(A)=0$. The resolution $(E, K)$ of $A$ given by (3.4) has $K_{s}$ core-equal and so $K_{s}$ is in the genus of an $A$-core. Suppose $B=K_{s} \oplus \mathbb{Z} G^{(m)}$ is in the genus of $A$. Adding $\mathbb{Z} G^{(m)}$ to $E_{s-1}$ and adjusting $(E, K)$ at all integers in $[s-1,1] \cap I(A)$ produces a partial free resolution as required.

Conversely, taking a projective excision of the given partial resolution shows

$$
\varepsilon_{s-1}[F]=\varepsilon_{s-1}[P]+\left[\mathbb{Z} G^{(m)}\right]
$$

where $P_{*}$ is the minimal projective summand of the excision. Hence $\varepsilon_{s-1}[P]$ $=0$ and so $\sigma_{s-1}(A)=0$.

\section{REMARKS.}

(1) If the genus of $A$ in (3.5) consists of only one isomorphism class then $\sigma_{s-1}(A)=0$ is a necessary and sufficient condition for the existence of a free resolution of period $s$. An example of this situation is, of course, $A=\mathbb{Z}$. This is Swan's old result.

(2) There are weaker conditions that still guarantee in (3.5) a free resolution of period $s$. Here is an example.

If, for every $B$ in the genus of $A$, there exists a projective module $P$ so that $A \oplus P \simeq B \oplus P$ and $\mathbb{Z} G$ allows cancellation, then $\sigma_{s-1}(A)=0$ implies $A$ has a periodic free resolution of period $s$.

Concerning the hypothesis on the genus of $A$, note that in general, $A$ and $B$ belong to the same genus if and only if there exist projectives $P$ and $Q$ 
in the same genus so that $A \oplus P \simeq B \oplus Q$ : for we may embed $B$ in $A$ with finite cokernel $M$ of order prime to $|G|, 0 \rightarrow B \rightarrow A \rightarrow M \rightarrow 0$, and then take a projective presentation of $M, 0 \rightarrow P \rightarrow Q \rightarrow M \rightarrow 0$.

The italicized assertion above is a consequence of Corollary (3.5) and the following result.

(3.6) LEMMA. Let $0 \rightarrow B \rightarrow \mathbb{Z} G^{(m)} \rightarrow L \rightarrow 0$ be a free presentation and assume $B \oplus \mathbb{Z} G^{(k)} \simeq A \oplus \mathbb{Z} G^{(k)}$. Then there always exists a free presentation

$$
0 \rightarrow A \oplus \mathbb{Z} G \rightarrow \mathbb{Z} G^{(m+1)} \rightarrow L \rightarrow 0 ;
$$

while if $\mathbb{Z} G$ allows cancellation or $m \geq 2$, there is a free presentation

$$
0 \rightarrow A \rightarrow \mathbb{Z} G^{(m)} \rightarrow L \rightarrow 0 .
$$

Proof. Since $B \oplus \mathbb{Z} G^{(2)}$ satisfies the Eichler condition, we obtain $B \oplus \mathbb{Z} G^{(2)} \simeq A \oplus \mathbb{Z} G^{(2)}$ by cancelling $\mathbb{Z} G^{(k-2)}$ (assuming $k \geq 2$ ) and hence a short exact sequence

$$
0 \rightarrow A \oplus \mathbb{Z} G^{(2)} \stackrel{\phi}{\rightarrow} \mathbb{Z} G^{(m+2)} \rightarrow L \rightarrow 0 .
$$

Let $P \oplus \mathbb{Z} G^{(2)} \phi=\mathbb{Z} G^{(m+2)}$. Then $P \oplus \mathbb{Z} G \phi$ is free since $m \geq 1$ so that $\mathbb{Z} G^{(m+1)}$ allows cancellation, while if $\mathbb{Z} G$ allows cancellation or $m \geq 2$, $P$ itself is free. Now $\phi$ induces an injection $A \rightarrow P$ whose cokernel is isomorphic to $L$.

\section{Periodic stably free resolutions}

We begin with some remarks on Poincaré series. Let $\phi$ and $\psi$ be $\mathbb{Z}$ valued functions defined on $\mathbb{Z}_{\geq 0}$. If there exists $N$ such that $\phi(n)=\psi(n)$ for all $n \geq N$ we shall say that $\phi$ and $\psi$ are ultimately equal. The function $\psi: \mathbb{Z}_{\geq 0} \rightarrow \mathbb{Z}$ is called PORC modulo $q$ (polynomial on residue classes modulo $q$ ) if there exist polynomials $f_{0}(t), \ldots, f_{q-1}(t)$ in $\mathbb{Q}[t]$ so that for $n=r q+i, 0 \leq i<q$, we have $\psi(n)=f_{i}(r)$. A function is called ultimately PORC if it ultimately equals a PORC function.

The Poincaré series associated with $\phi$ is the formal power series

$$
P(\phi)=\sum_{n \geq 0} \phi(n) t^{n},
$$

and it is a well-known fact that $\phi$ is ultimately PORC modulo $q$ if and only if $P(\phi)$ is a rational function of the form

$$
P(\phi)=\frac{g(t)}{\left(1-t^{q}\right)^{m}},
$$


where $g(t) \in \mathbb{Z}[t]$ and $m$ is a positive integer. Note that if $\phi$ is periodic with period $s$ (meaning that $\phi(n+s)=\phi(n)$ for all $n \geq 0$ ) then

$$
P(\phi)=\frac{\phi(0)+\phi(1) t+\cdots+\phi(s-1) t^{s-1}}{1-t^{s}} .
$$

As was noted in the introduction to this paper, for every $\mathbb{Z} G$-lattice $A$, the function $\varepsilon\left(A_{(G)}\right)$ is ultimately PORC. We now claim the same of the function $\varepsilon(A)$.

(4.1) TheOrem. If $A$ is a $\mathbb{Z} G$-lattice, then $\varepsilon(A)$ is ultimately PORC.

When $A$ is non-periodic we already effectively know the result: by (2.7) and (3.1), $\varepsilon(A)$ is ultimately equal to $\varepsilon\left(A_{(G)}\right)$, whence $\varepsilon(A)$ is ultimately PORC because $\varepsilon\left(A_{(G)}\right)$ is. There remains the periodic case and this is a consequence of the following result.

(4.2) Proposition. If the $\mathbb{Z} G$-lattice $A$ is periodic with projective period $q$, then $\varepsilon(A)$ is periodic with period a multiple of $2 q$.

Proof. We may work with twice the projective period and so shall assume without loss of generality that $q$ is even. Let $A=A^{\prime} \oplus Q$ be a projective excision and $\left(P^{\prime}, C\right)$ a periodic minimal projective resolution of $A^{\prime}$. If $(P, C)$ is the projective resolution of $A$ which coincides with $\left(P^{\prime}, C\right)$ at all positive dimensions but has $P_{0}=P_{0}^{\prime} \oplus Q$, then $(P, C)$ is a minimal projective resolution of $A$. For any $0<i<q$ and $k>0$ we have $P_{k q+i}=P_{i}$ and $P_{k q}=P_{q}=P_{0}^{\prime}$. Hence if $0 \leq i<q$ and $k>0$,

$$
\begin{aligned}
\varepsilon_{k q+i}[P]= & {\left[P_{i}\right]-\left[P_{i-1}\right]+\cdots+(-1)^{i}\left[P_{0}^{\prime}\right] } \\
& +(-1)^{i+1} k\left\{\left[P_{q-1}\right]-\cdots+(-1)^{q-1}\left[P_{0}^{\prime}\right]\right\}+(-1)^{i+1+q-1}[Q]
\end{aligned}
$$

and $(-1)^{i+q}=(-1)^{i}$ because $q$ is even. Let $e$ be the exponent of $K(\mathbb{Z} G)$ (recall that the projective class group is finite). Then for all $m>0$,

$$
\begin{aligned}
\varepsilon_{m e q+i}[P] & =\left[P_{i}\right]-\left[P_{i-1}\right]+\cdots+(-1)^{i-1}\left[P_{1}\right]+(-1)^{i}\left[P_{0}^{\prime} \oplus Q\right] \\
& =\varepsilon_{i}[P] .
\end{aligned}
$$

Now $S_{k q+j}(A)=S_{j}(A)$ for all $j>0$ and $k>0$ and therefore $\varepsilon_{i}[P] \epsilon$ $S_{i+1}(A)$ if and only if $\varepsilon_{m e q+i}[P] \in S_{m e q+i+1}(A)$. Thus $i \in I(A)$ if and only if $m e q+i \in I(A)$ for all $m>0$ and $0 \leq i<q$. Since $\varepsilon\left(A_{(G)}\right)$ is periodic of period $q$, it now follows (by (3.1)) that $\varepsilon(A)$ is periodic of period $e q$.

EXAMPLE. Let $G$ be cyclic of prime order and chosen so that $K(\mathbb{Z} G) \neq$ 0 . We may therefore find a non-free projective ideal $Q$ in $\mathbb{Z} G$. Then 
$\varepsilon\left(Q_{(G)}\right)=(1,-1,1,-1, \ldots)$ and $\varepsilon(Q)=(2,0,2,0, \ldots)$, whence $I(Q)$ is empty. The usual minimal free resolution of $\mathbb{Z}$ of period 2 is also minimal projective and so $\varepsilon(\mathbb{Z})=\varepsilon\left(\mathbb{Z}_{(G)}\right)=(1,0,1,0, \ldots)$ and $I(\mathbb{Z})=\mathbb{Z}_{\geq 0}$. The kernels in this resolution are $\mathbb{Z}$ and $\mathfrak{g}$, the augmentation ideal. Now $S_{0}(\mathbb{Z})=$ $C_{\mathbb{Q}}=0$ and $S_{1}(\mathbb{Z})=C_{\mathbb{Q g}_{\mathfrak{g}}}=K(\mathbb{Z} G)$. Letting $A=\mathbb{Z} \oplus Q$, we see from the calculation in the proof of (4.2) that $\sigma_{i}(A) \in S_{i+1}(A)$ if and only if $[Q] \in S_{i+1}(A)$. Thus $I(A)=2 \mathbb{Z}_{\geq 0}$ and $\varepsilon\left(A_{(G)}\right)=(2,-1,2,-1, \ldots)$, while $\varepsilon(A)=(2,0,2,0, \ldots)$.

(4.3) Theorem. If $A$ is a periodic $\mathbb{Z} G$-lattice, then there exists a stably free resolution $(E, K)$ of $A$ which is ultimately periodic of period a multiple of the projective period of $A$ and has all the properties of the resolution in Theorem 3.4 .

Proof. Let $(E, K)$ be the stably free resolution of (3.4). By (4.2), $\varepsilon(A)$ is periodic of some period $r$, an even multiple of the projective period of $A$. Since $\varepsilon\left(A_{(G)}\right)$ also has period $r$,

$$
m \in I(A) \Leftrightarrow m+r \in I(A) .
$$

Let $(P, C)$ be a minimal projective resolution of $A$ occurring in a projective excision of $(E, K)$. If $m \in I(A)$, then $K_{m+1}=C_{m+1}$ while if $m \notin I(A)$, then $K_{m+1}$ is in the genus of $C_{m+1} \oplus \mathbb{Z} G$. The lattices $C_{r}, C_{2 r}, \ldots$ all belong to a single genus and therefore, using also (1), the lattices $K_{r}, K_{2 r}, \ldots$ belong to a single genus. By the Jordan-Zassenhaus theorem we can find $m<n$ so that $K_{m r} \simeq K_{n r}$. Replace ${ }_{\infty}(E, K)_{n r}$ by repeats of the segment $[n r-1, m r)$ and call the resulting resolution $E_{*}^{\prime}$.

Now

$$
0 \rightarrow K_{n r} \rightarrow E_{n r-1} \rightarrow \cdots \rightarrow E_{m r} \rightarrow K_{m r} \rightarrow 0
$$

is exact and so, because $(n-m) r$ is even,

$$
e_{n r-1}-e_{n r-2}+\cdots-e_{m r}=0,
$$

where $e_{i}=\operatorname{pr} E_{i}$. Hence if $0 \leq i<(n-m) r$ and $k \geq 0$,

$$
\varepsilon_{n r+i+k(n-m) r}\left(E_{(G)}^{\prime}\right)=\varepsilon_{m r+i}\left(E_{(G)}^{\prime}\right) \text {. }
$$

But

$$
\begin{aligned}
\varepsilon_{m r+i}\left(E_{(G)}^{\prime}\right) & =\varepsilon_{m r+i}\left(E_{(G)}\right) & & \text { (by construction) } \\
& =\varepsilon_{m r+i}(A) & & (\text { by }(3.4)) \\
& =\varepsilon_{n r+i+k(n-m) r}(A) & & (\text { by }(4.2)) .
\end{aligned}
$$


Thus $E_{*}^{\prime}$ is a stably free resolution with the four properties of (3.4) and it is periodic beyond $m r$ with period $(n-m) r$.

\section{Appendix 1}

Proposition. Given a positive integer $N$, there exist a group $G$ and $a$ $\mathbb{Z} G$-lattice $A$ having projective period $N$.

Proof. In the arithmetic sequence $1+k N$ choose an odd prime $p$. Find $1<r<p$ such that $r$ has order $N \bmod p$. Let $A=\langle a\rangle$ be cyclic of order $p, B=\langle b\rangle$ be cyclic of order $N$ and let $B$ act on $A$ by $b^{-1} a b=a^{r}$. If $G$ denotes the split extension of $A$ by $B$, then the trivial $\mathbb{Z} G$-module $\mathbb{Z}$ has projective period $2 N$ (e.g. [6]). This proves the proposition for all even integers.

Henceforth assume $N$ is odd and take a periodic minimal projective resolution $(P, C)$ of $\mathbb{Z}$. Then

$$
0 \rightarrow C_{N} \oplus C_{2 N} \rightarrow P_{N-1} \oplus P_{2 N-1} \rightarrow \cdots \rightarrow P_{0} \oplus P_{N} \rightarrow \mathbb{Z} \oplus C_{N} \rightarrow 0
$$

is exact and $C_{2 N}=\mathbb{Z}$. Hence $\mathbb{Z} \oplus C_{N}$ is periodic with $N$ a period. We claim the exact (projective) period is $N$. Suppose not and that the projective period is $l<N$. Then $l$ divides $N$ (otherwise the greatest common divisor of $l$ and $N$ is a period and is strictly smaller than $l$ ) and so $l$ is also odd. Now

$$
\operatorname{Ext}_{\mathbf{Z} G}^{i}(\mathbb{Z},) \oplus \operatorname{Ext}_{\mathbf{Z} G}^{i}\left(C_{N},\right) \simeq \operatorname{Ext}_{\mathbb{Z} G}^{i+l}(\mathbb{Z},) \oplus \operatorname{Ext}_{\mathbb{Z} G}^{i+l}\left(C_{N},\right),
$$

whence

$$
H^{i}(G,) \oplus H^{i+N}(G,) \simeq H^{i+l}(G,) \oplus H^{i+l+N}(G,) .
$$

We know that $H^{i}(G, \mathbb{Z})=0$ for all odd $i$. So with $i$ even in formula (1),

$$
H^{i}(G, \mathbb{Z}) \simeq H^{i+l+N}(G, \mathbb{Z}) .
$$

The projective period of $\mathbb{Z}$ is the smallest $k$ such that $H^{i}(G, \mathbb{Z})$ is cyclic of order $|G|$ [4, Lemma 4.2]. Taking $i=N-l$ in (2) gives

$$
H^{N-l}(G, \mathbb{Z}) \simeq H^{2 N}(G, \mathbb{Z}) \simeq \widehat{H}^{0}(G, \mathbb{Z}) \quad \text { (Tate cohomology) }
$$

and therefore $N-l$ is the projective period of $\mathbb{Z}$. This is a contradiction, whence $l=N$ and the Proposition is proved.

\section{Appendix 2}

The decomposition theorem of [2] is the following result. 
TheOREM. Suppose $(P, C)$ is a minimal projective resolution of the $\mathbb{Z} G$ lattice $A$. Then $C_{n+1}$ decomposes as

$$
C_{n+1}=D_{1} \oplus \cdots \oplus D_{k}
$$

with all $D_{i} \neq 0$ if and only if there exists a decomposition

$$
A \oplus \mathbb{Z} G^{(t)}=L_{1} \oplus \cdots \oplus L_{k}
$$

for some $t \geq 0$, with all $L_{i}$ non-projective, and satisfying

$$
\chi_{n}\left(A \oplus \mathbb{Z} G^{(t)}\right)=\chi_{n}\left(L_{1}\right)+\cdots+\chi_{n}\left(L_{k}\right)
$$

The difference between the above statement and (7.1) of [2] is that in (7.1), $A$ is restricted to a special class of lattices. We claim that this restriction is unnecessary.

The sufficiency part of the theorem as given in [2, Theorem (5.1)] is valid for all lattices. There remains the necessity. The following argument is due to Serge Aloneftis. We shall write $\Lambda=\mathbb{Z}_{(G)} G$.

Lemma. Let $(E, K)$, be a $\Lambda$-projective resolution of the $\Lambda$-lattice $V$ and assume

$$
K_{n+1}=M_{1} \oplus \cdots \oplus M_{k}
$$

with all $M_{i} \neq 0$. Then there exists $t \geq 0$ such that

$$
V \oplus \Lambda^{(t)}=N_{1} \oplus \cdots \oplus N_{k}
$$

and projective resolutions $\left({ }_{i} E,{ }_{i} K\right)$ of $N_{i}(1 \leq i \leq k)$ satisfying

$$
\chi_{n}(\widetilde{E})=\chi_{n}\left({ }_{1} E\right)+\cdots+\chi_{n}\left({ }_{k} E\right),
$$

where $\widetilde{E}_{i}=E_{i}$ for all $i>0$ and $\widetilde{E}_{0}=E_{0} \oplus \Lambda^{(t)}$.

Proof. For a given $\Lambda$-lattice $W$ we denote its $\mathbb{Z}_{(G)}$-dual $\operatorname{Hom}_{\mathbb{Z}_{(G)}}\left(W, \mathbb{Z}_{(G)}\right)$ by $W^{*}$. For each $i=1, \ldots, k$, choose a projective resolution $\left({ }_{i} F,{ }_{i} B\right)$ of $M_{i}^{*}$. The direct sum of these gives a projective resolution of

$$
M_{1}^{*} \oplus \cdots \oplus M_{k}^{*} \simeq K_{n+1}^{*}
$$

and hence Schanuel's Lemma, applied to this resolution and the dual of $(E, K)$, both truncated at dimension $n$, yields

$$
V^{*} \oplus \Lambda^{(t)} \simeq{ }_{1} B_{n+1} \oplus \cdots \oplus{ }_{k} B_{n+1} \oplus \Lambda^{(s)}
$$

for suitable $t, s$ in $\mathbb{Z}_{\geq 0}$.

The dual of ${ }_{n}\left({ }_{i} F,{ }_{i} B\right)_{0}$ is

$$
0 \rightarrow M_{i} \rightarrow{ }_{i} F_{0} \rightarrow \cdots \rightarrow{ }_{i} F_{n} \rightarrow{ }_{i} B_{n+1}^{*} \rightarrow 0 \text {. }
$$


When $i<k$, we view (1) as the $n$th truncation of a projective resolution $\left({ }_{i} E,{ }_{i} K\right)$; while $\left({ }_{k} E,{ }_{k} K\right)$ is to be a projective resolution whose $n$th truncation is (1) but with $\Lambda^{(s)}$ added to ${ }_{k} F_{n}$ and ${ }_{k} B_{n+1}^{*}$. If we set $N_{i}={ }_{i} B_{n+1}^{*}$ for $i<k$ and $N_{k}={ }_{k} B_{n+1}^{*} \oplus \Lambda^{(s)}$, then $\bigoplus_{i=1}^{k}\left({ }_{i} E,{ }_{i} K\right)$ is a projective resolution of $N_{1} \oplus \cdots \oplus N_{k} \simeq V \oplus \Lambda^{(t)}$, with $(n+1)$ st kernel $M_{1} \oplus \cdots \oplus M_{k}$. Since $(\widetilde{E}, K)$ is also a projective resolution of $V \oplus \Lambda^{(t)}$, Schanuel's Lemma yields

$$
K_{n+1} \oplus \Lambda^{(e)} \simeq M_{1} \oplus \cdots \oplus M_{k} \oplus \Lambda^{(f)}
$$

for suitable $e, f$ in $\mathbb{Z}_{\geq 0}$. Semi-local cancellation gives $e=f$, which is exactly the required equality

$$
\chi_{n}(\widetilde{E})=\chi_{n}\left({ }_{1} E\right)+\cdots+\chi_{n}\left({ }_{k} E\right) .
$$

We return to the theorem and assume $C_{n+1}$ decomposes. The minimality of $(P, C)$ ensures that $C_{n+1}$ is core-equal, whence so is each summand $D_{i}$. Apply the lemma with $V \stackrel{n+1}{=} A_{(G)},(E, K)=(P, C)_{(G)}$ and $M_{i}=\left(D_{i}\right)_{(G)}$. Because $\widetilde{E}$ is a minimal projective resolution of $V \oplus \Lambda^{(t)}$,

$$
\chi_{n}(\widetilde{E})=\chi_{n}\left(V \oplus \Lambda^{(t)}\right)=\chi_{n}\left(A \oplus \mathbb{Z} G^{(t)}\right) .
$$

Let $A \oplus \mathbb{Z} G^{(t)}=L_{1} \oplus \cdots \oplus L_{k}$ be a decomposition with $\left(L_{i}\right)_{(G)} \simeq N_{i}$ for all $i$. Since $M_{i}$ is core-equal, a projective excision of ${ }_{i} E$ shows $\chi_{n}\left({ }_{i} E\right)=\chi_{n}\left(N_{i}\right)$ and so $\chi_{n}\left({ }_{i} E\right)=\chi_{n}\left(L_{i}\right)$. The conclusion of the lemma is now the required equality for $\chi_{n}$ in the theorem.

Linnell's theorem (6.1) in [2] is thus not needed for the decomposition theorem. Of course, it comes in crucially when we search for conditions under which $A \oplus \mathbb{Z} G^{(t)}$ decomposes non-projectively. If $\mathscr{Z}$ (as in [2]) is the class of all $\mathbb{Z} G$-lattices that occur as direct summands of kernels (including the 0th kernel) in projective resolutions of $\mathbb{Z} \oplus \mathbb{Z} G^{(t)}$ for all $t \geq 0$, then for every $A$ in $\mathscr{Z}, A \oplus$ free decomposes non-projectively if and only if $\pi(A)$ is a disjoint union of closed subsets of $\pi(G)$.

Are there similar graphical criteria for general $A$ ? Moreover, do there exist sensible combinatorial conditions on $G$ that ensure the additivity of $\chi_{n}$ on a non-projective decomposition?

\section{References}

[1] H. Bass, Algebraic $K$-theory (Benjamin, New York, 1968).

[2] K. W. Gruenberg, 'Partial Euler characteristics of finite groups and the decomposition of lattices', Proc. London Math. Soc. (3) 48 (1984), 91-107. 
[3] K. W. Gruenberg and P. A. Linnell, 'Minimal free resolutions of lattices over finite groups', Illinois J. Math. 32 (1988), 361-374.

[4] R. G. Swan, 'Periodic resolutions for finite groups', Ann. of Math. 72 (1960), 267-291.

[5] R. G. Swan, 'Minimal resolutions for finite groups', Topology 4 (1965), 193-208.

[6] O. Talelli, 'On minimal resolutions for metacyclic groups with periodic cohomology', Comm. Algebra 12 (1984), 1343-1360.

[7] P. J. Webb, 'Bounding the ranks of $\mathbb{Z} G$-lattices by their restrictions to elementary abelian subgroups', J. Pure Appl. Algebra 23 (1982), 311-318.

Queen Mary and Westfield College

London

England 